\title{
Influence of low-order room reflections on sound zone system performance
}

M. Olik, P. J. B. Jackson and P. D. Coleman

CVSSP, Department of Electronic Engineering, University of Surrey, UK

$22 / 01 / 2012$

\begin{abstract}
Studies on sound field control methods able to create independent listening zones in a single acoustic space have recently been undertaken due to the potential of such methods for various practical applications, such as individual audio streams in home entertainment. Existing solutions to the problem have shown to be effective in creating high and low sound energy regions under anechoic conditions. Although some case studies in a reflective environment can also be found, the capabilities of sound zoning methods in rooms have not been fully explored. In this paper, the influence of low-order (early) reflections on the performance of key sound zone techniques is examined. Analytic considerations for small-scale systems reveal strong dependence of performance on parameters such as source positioning with respect to zone locations and room surfaces, as well as the parameters of the receiver configuration. These dependencies are further investigated through numerical simulation to determine system configurations which maximize the performance in terms of acoustic contrast and array control effort. The design rules for source and receiver positioning are suggested, for improved performance under a given set of constraints such as a number of available sources, zone locations and the direction of the dominant reflection.
\end{abstract}




\section{INTRODUCTION}

The sound zoning problem has been of interest to the acoustic research community for over a decade. The main aim of a sound zoning system is to create acoustic separation between regions in a space, to allow for listening to different audio streams in that space without the need for headphones. Potential applications of such a system include car audio, multi-player computer gaming and audio for public exhibitions.

The existing sound zoning solutions fall into two main categories: sound energy control (acoustic contrast and brightness control [1], acoustic energy difference maximization [2], sound power minimization [3]) and sound field synthesis methods (analytical [4] and based on measured system responses [5]). Hybrid techniques [6, 7] and crosstalk cancellation [8] have also been applied to the problem. Compared to the synthesis methods, the energy control techniques can achieve high acoustic contrast between two anechoic regions with small source arrays [9], as shown in [10, 11]. Jones and Elliott extended the investigations into near-field reflective sound field in a case study [3], in which array geometry w.r.t (with respect to) the zones was adjusted empirically for improved contrast.

Here, we seek to provide a more general insight into reflective performance through analytical description of the far-field performance of a simple 2-loudspeaker, 2-receiver $(2 \times 2)$ system with a single reflector. Expressions for the key performance measures, acoustic contrast and control effort, are derived for a representative method (acoustic contrast control) to provide guidelines for geometrical adjustments to alleviate the effects of reflection. The contrast and effort performance of a geometrically optimized $2 \times 2$ system is evaluated in numerical simulations. Differences between the performance characteristics under matched and mismatched setup conditions are observed. The generalizability of the results is tested on $3 \times 4$ and $4 \times 18$ configurations.

\section{THEORY}

In the following section, the source weight calculation procedure and evaluation metrics for the acoustic contrast control (ACC) method are described. The mathematical expressions for acoustic contrast and control effort of a $2 \times 2$ ACC system with a single reflector are derived under matched and mismatched setup/playback conditions.

\section{Acoustic Contrast Control (ACC) Method}

ACC is a sound energy control method that aims to maximize energy in one region (bright zone A) while minimizing it in another (dark zone B), by maximizing the following cost function [1]:

$$
C=\frac{N_{B s} \mathbf{p}_{A s}^{H} \mathbf{p}_{A s}}{N_{A s} \mathbf{p}_{B s}^{H} \mathbf{p}_{B s}}=\frac{N_{B s} \mathbf{q}^{H} \mathbf{G}_{A s}^{H} \mathbf{G}_{A s} \mathbf{q}}{N_{A s} \mathbf{q}^{H} \mathbf{G}_{B s}^{H} \mathbf{G}_{B s} \mathbf{q}}
$$

where $\mathbf{p}_{A s}$ and $\mathbf{p}_{B s}$ are the vectors of complex harmonic pressures at setup receiver locations in the zones (subscripts $A$ and $B$ denote the bright and dark zone, respectively, and $s$ denotes a setup receiver), $N_{A s}$ and $N_{B s}$ are the numbers of setup receivers in the zones, $\mathbf{q}$ is the vector of complex source strengths, $\mathbf{G}_{A s}$ and $\mathbf{G}_{B s}$ are the matrices of responses between the sources and setup receivers, and $H$ denotes conjugate transpose. Choi and Kim [1] showed that the vector $\mathbf{q}_{\text {opt }}$ which maximizes $C$ is proportional to the eigenvector of the matrix $\left[\mathbf{G}_{B s}^{H} \mathbf{G}_{B s}\right]^{-1} \mathbf{G}_{A s}^{H} \mathbf{G}_{A s}$ that corresponds to its largest eigenvalue. When the number of setup receivers in the dark zone is less than the number of sources, the matrix $\mathbf{G}_{B s}^{H} \mathbf{G}_{B s}$ becomes singular. Analytical solution in this case is possible by adding a regularization parameter $\beta$ to the main diagonal of this matrix, so the matrix for EVD (eigenvalue decomposition) becomes $\left[\mathbf{G}_{B s}^{H} \mathbf{G}_{B s}+\beta \mathbf{I}\right]^{-1} \mathbf{G}_{A s}^{H} \mathbf{G}_{A s}$. 
The acoustical separation between the bright and dark zones can be quantified:

$$
\text { contrast }=10 \log _{10}\left(\frac{N_{B m} \mathbf{q}_{o p t}^{H} \mathbf{G}_{A m}^{H} \mathbf{G}_{A m} \mathbf{q}_{o p t}}{N_{A m} \mathbf{q}_{o p t}^{H} \mathbf{G}_{B m}^{H} \mathbf{G}_{B m} \mathbf{q}_{\text {opt }}}\right)
$$

where the subscript $m$ denotes a monitor receiver location. Distinguishing the points for system setup and performance evaluation is important for ensuring independence, especially when only one set of responses (from one measurement run) is available, or when analytical responses are used. Performance predictions based on the setup points lead to overestimated contrast that is never attainable in practice [12].

Our second performance metric is control effort, which is defined as

$$
\text { effort }=10 \log _{10}\left(\eta^{2} \mathbf{q}_{\text {opt }}^{H} \mathbf{q}_{\text {opt }}\right)-10 \log _{10}\left|q_{\text {ref }}\right|^{2}
$$

where $\eta=\frac{p_{0}}{\bar{p}_{A s}} 10^{L_{t} / 20}$ is a sound pressure level compensation factor required to obtain a frequency independent SPL in the bright zone, with $p_{0}=2 \cdot 10^{-5} \mathrm{~Pa}$ as the reference pressure, $\bar{p}_{A s}$ as the rms pressure in the bright zone before compensation and $L_{t}$ denoting a target level; $q_{\text {ref }}$ is the source strength of a reference monopole generating the target SPL in the bright zone.

\section{Acoustic Contrast and Control Effort Expressions: $2 \times 2$ system}

The mathematical expressions for contrast and effort of a $2 \times 2$ ACC system are derived below. The limitation in degrees of freedom does not differentiate this solution from other methods (e.g. crosstalk cancellation [13]). Two cases are considered: system setup in anechoic conditions and playback in reflective conditions (condition 1), and setup and playback both in reflective conditions (condition 2). Condition 1 is important from the point of view of a practical system: in some situations responses from the playback room may not be available for source weight calculation. The derivations for the setup stage of condition 1 are completed assuming the freefield Green's transfer function between a source and receiver, with point monopole sources and omnidirectional pressure receivers:

$$
\mathbf{G}^{(1)}=\mathbf{A D}
$$

where $\mathbf{D}=e^{-j k r} / r$ in which $r$ denotes the distance between a source and receiver, $\mathbf{A}=j \rho c k / 4 \pi$ is the complex amplitude in which $\rho$ is the density of air, $c$ is the speed of sound and $k=\omega / c$ is the wave number. For condition 2 and the playback stage of condition 1, the derivations are based on the assumptions of the image source model (ISM). When only a single reflection is considered, the free-field response is modified to read:

$$
\mathbf{G}^{(2)}=\mathbf{A}\left(\mathbf{D}+\gamma \mathbf{D}^{\prime}\right)
$$

where $\mathbf{D}^{\prime}=e^{-j k r^{\prime}} / r^{\prime}$, in which $r^{\prime}$ denotes the distance between an image source and a receiver and $\gamma=\sqrt{1-\alpha}$ is the magnitude of the reflection coefficient of the surface characterized by absorption coefficient $\alpha$.

The following expressions are derived using the far-field approximation: $d \ll r$ and $d \ll r^{\prime}$, where $d$ is the spacing between the sources (see Fig. 1 (a)). The assumption does not differentiate between the distances $\left(r_{1}, r_{2}\right)$ and $\left(r_{1}^{\prime}, r_{2}^{\prime}\right)$ in the amplitude terms of the equations, replacing each pair with distances from the center of array $r$ and $r^{\prime}$, respectively. This allows for a significant simplification of the analysis. In practical situations, the far-field approximation applies when the zones are located at some distance from a loudspeaker array. In the derivation of control effort expressions it is assumed that the reference monopole is located at the center of the source array. The EVD matrix used to obtain the solution is regularized, but the regularization parameter $\beta$ is set so as to be negligible. Fig. 1 (b) shows the configuration used in the analysis. 
(a)

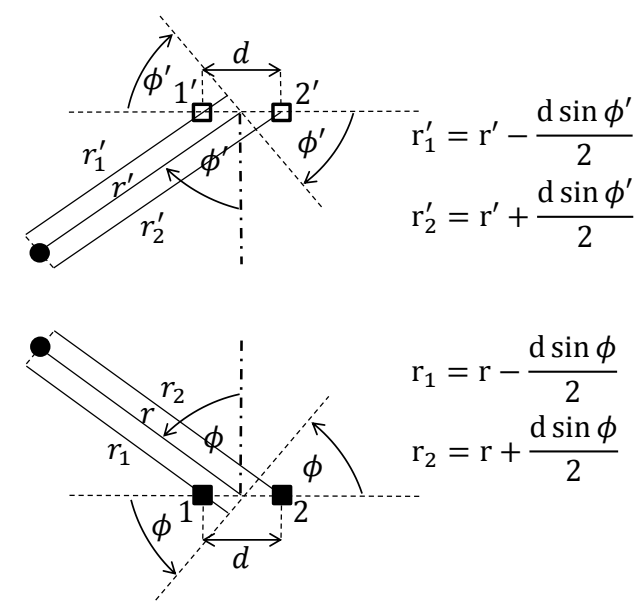

(b)

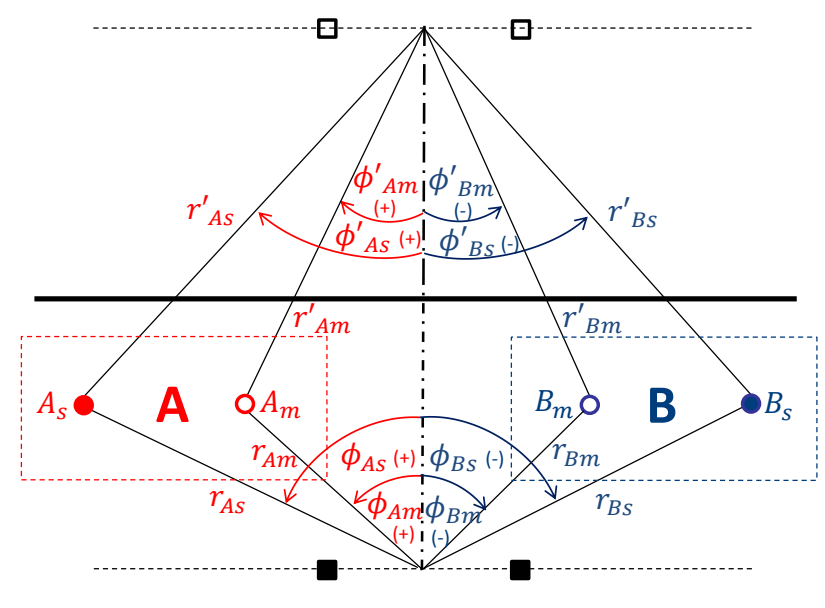

Figure 1: (Color online) A $2 \times 2$ system under analysis (not to scale): (a) far-field approximation, (b) system diagram. Symbol assignment: $\square$ source, $\square$ image source, - setup receiver, o monitor receiver, - surface; $\phi$ denotes an angle between array or image array axis (dash-dotted line) and the line between the center of array and a given receiver (thin solid line). (+) and (-) indicate positive and negative angles, respectively.

The analysis shows that the magnitude of acoustic pressure in each zone consists of three main components: 0th order component due to direct sound cancellation, 1st order component due to playback in the reflective environment and 1st order components due to system setup in the reflective environment, where the order is with respect to parameter $\gamma$. These components yield three combinations and all six components are listed in Table 1. Higher order components and related combinations did not have a major influence on contrast and effort, so we have discarded them from further analysis.

Assuming $N=N_{A m}=N_{B m}$, the acoustic contrast expressions for conditions 1 and 2 are

$$
\begin{gathered}
\text { contrast }_{A B}^{(1)} \approx 10 \log _{10}\left(\frac{\sum_{i=1}^{N}\left(P_{A m D i r_{i}}^{2}+P_{A m R 1_{i}}^{2}+P_{A m C 1_{i}}\right)}{\sum_{i=1}^{N}\left(P_{B m D i r_{i}}^{2}+P_{B m R 1_{i}}^{2}+P_{B m C 1_{i}}\right)}\right) \\
\text { contrast }_{A B}^{(2)} \approx 10 \log _{10}\left(\frac{\sum_{i=1}^{N}\left(P_{A m D i r_{i}}^{2}+P_{A m R 1_{i}}^{2}+P_{A m R 2_{i}}^{2}+P_{A m C 1_{i}}+P_{A m C 2_{i}}+P_{A m C 3_{i}}\right)}{\sum_{i=1}^{N}\left(P_{B m D i r_{i}}^{2}+P_{B m R 1_{i}}^{2}+P_{B m R 2_{i}}^{2}+P_{B m C 1_{i}}+P_{B m C 2_{i}}+P_{B m C 3_{i}}\right)}\right)
\end{gathered}
$$

Under condition 2 , the expression for control effort of the $2 \times 2$ system is

$$
\begin{aligned}
\text { effort }_{A}^{(2)} & \approx 10 \log _{10}\left(\frac{1}{\left(r_{B s} r_{A s}\right)^{2}}+\frac{\gamma^{2}}{\left(r_{B s}^{\prime} r_{A s}\right)^{2}}+\frac{2 \gamma}{r_{B s} r_{B s}^{\prime} r_{A s}^{2}} \cos \left(\frac{k d\left(\sin \phi_{B s}-\sin \phi_{B s}^{\prime}\right)}{2}\right) \cos \left(k\left(r_{B s}-r_{B s}^{\prime}\right)\right)\right) \\
& -10 \log _{10}\left(P_{A s D i r}^{2}+P_{A s R 1}^{2}+P_{A s R 2}^{2}+P_{A s C 1}+P_{A s C 2}+P_{A s C 3}\right)-3
\end{aligned}
$$

With these analytical expressions, we can seek to explain the behaviors of various $2 \times 2$ configurations which we explore in the following simulations.

\section{Sound Zone Performance with a Single Reflection - Analysis}

In this section, means of improving acoustic contrast performance of a $2 \times 2$ system by adjusting system geometry are explored. The analysis is based on Eqs. 6 and 7. Two geometrical design rules for reducing the effect of a reflection on contrast are suggested and the related control effort is analyzed. The optimized geometries are tested in numerical simulations for $2 \times 2,3 \times 4$ and $4 \times 18$ systems. Performance effects are demonstrated with the configurations drawn in Fig. 2 . 
TABLE 1: Components of the magnitude of sound pressure generated by a $2 \times 2$ system based on the ACC method, $X \in\{A m, B m, A s, B s\}, \Delta_{X D i r}=\sin \phi_{X}-\sin \phi_{B s}, \Delta_{X R 1}=\sin \phi_{X}^{\prime}-\sin \phi_{B s}$ and $\Delta_{X R 2}=\sin \phi_{X}-\sin \phi_{B s}^{\prime}$. The order is with respect to $\gamma$.

\begin{tabular}{lcc}
\hline Definition & Order & Origin \\
\hline$P_{X D i r}=\sin \left(k d \Delta_{X D i r} / 2\right) / r_{X} r_{B s}$ & 0 & Direct Setup \\
$P_{X R 1}=\gamma \sin \left(k d \Delta_{X R 1} / 2\right) / r_{X}^{\prime} r_{B s}$ & 1 & Reflective Playback \\
$P_{X R 2}=\gamma \sin \left(k d \Delta_{X R 2} / 2\right) / r_{X} r_{B s}^{\prime}$ & 1 & Reflective Setup \\
$P_{X C 1}=2 P_{X D i r} P_{X R 1} \cos \left(k\left(r_{X}-r_{X}^{\prime}\right)\right)$ & 1 & Reflective Playback \\
$P_{X C 2}=2 P_{X D i r} P_{X R 2} \cos \left(k\left(r_{B s}-r_{B s}^{\prime}\right)\right)$ & 1 & Reflective Setup \\
$P_{X C 3}=2 P_{X R 1} P_{X R 2} \cos \left(k\left(r_{X}-r_{X}^{\prime}-r_{B s}+r_{B s}^{\prime}\right)\right)$ & 2 & Reflective Setup and Playback \\
\hline
\end{tabular}

\section{Optimization of System Geometry - Discussion}

According to Eq. 2, acoustic contrast can be improved by increasing the energy in the bright zone, decreasing the energy of the dark zone, or both. From the comparison with other sound energy control methods, it is known that ACC provides higher contrast by canceling the dark zone energy, rather than accumulating energy in the bright zone [3]. Thus, for the best contrast, we seek geometries that cancel well. To minimize the amount of sound energy in the dark zone, the reflected energy must be directed away. Hence, considering image sources, the reflected energy can be reduced in the dark zone by directing the image array's null towards the zone. This can be achieved by geometries for which a straight line between the center of array and the chosen dark-zone setup receiver creates a right angle with the surface. This requirement is not fulfilled by configuration I (see Fig. 2, left). To fix this, a reorientation of the sources and zones w.r.t. the surface is required, as in configuration II for example. The relationships $\phi_{B s}=\phi_{B s}^{\prime}$ and $\phi_{B s} \approx \phi_{B m} \approx \phi_{B m}^{\prime}$ are observed, and so the differences $\Delta_{B m R 1}=\sin \phi_{B m}^{\prime}-\sin \phi_{B s}$ and $\Delta_{B m R 2}=\sin \phi_{B m}-\sin \phi_{B s}^{\prime}$ contained in the reflective pressure components (see Table 1) are small. This minimizes the magnitude of $P_{B m R 1}$ and $P_{B m R 2}$ with the related combinations $P_{B m C 1}$, $P_{B m C 2}$ and $P_{B m C 3}$. According to Eq. 7, the overall sound energy in the dark zone is therefore reduced. Configuration III in Fig. 2 shows how to achieve further mitigation of the effect of the reflector: in addition to system reorientation w.r.t. the surface, the array is rotated around its central pivot so that $\phi_{B s}^{\prime}=-90^{\circ}$ and $\phi_{B m}^{\prime} \approx-90^{\circ}$. Increasing these angles causes a further drop in $\Delta_{B m R 1}$ and $\Delta_{B m R 2}$. Moreover, since $\phi_{B s}=-90^{\circ}$ and $\phi_{B m} \approx-90^{\circ}$, the direct sound component $P_{B m D i r}$ is also reduced due to decreasing $\Delta_{B m D i r}=\sin \phi_{B m}-\sin \phi_{B s}$.

It is also of interest to consider the geometries' control effort. To keep the effort low, the second term of Eq. 8 must maintain three large components $\Delta_{A s D i r}=\sin \phi_{A s}-\sin \phi_{B s}$ (criterion 1 - efficient direct sound cancellation), $\Delta_{A s R 1}=\sin \phi_{A s}^{\prime}-\sin \phi_{B s}$ (criterion 2 - lower effort due to addition of reflected energy in the bright zone) and $\Delta_{A s R 2}=\sin \phi_{A s}-\sin \phi_{B s}^{\prime}$ (criterion 3 - efficient reflected energy cancellation). The pairs of angles $\left(\phi_{A s}\right.$ and $\left.\phi_{B s}\right),\left(\phi_{A s}^{\prime}\right.$ and $\left.\phi_{B s}\right)$ and $\left(\phi_{A s}\right.$ and $\left.\phi_{B s}^{\prime}\right)$ must therefore be equal, with opposite signs, and close to $\pm 90^{\circ}$. To fulfill criterion 1 , the sources should ideally be placed equidistant between the zones, in a broadside configuration (to allow equal $\phi_{A s}$ and $\phi_{B s}$, with opposite signs) and close to the space between the two zones (for $\phi_{A s} \approx 90^{\circ}$ and $\phi_{B s} \approx-90^{\circ}$ ). Configurations I and II meet this requirement better than configuration III. The angle match required by criteria 2 and 3 is approximated by configuration I thanks to broadside orientation w.r.t. the surface. Configuration II fulfills criterion 3 better than configuration I, however at the cost of criterion 2. Configuration III meets criteria 2 and 3 poorly due to the rotation of sources. We can therefore expect large effort from configuration III, whereas for other two configurations the effort should remain low at similar levels. 

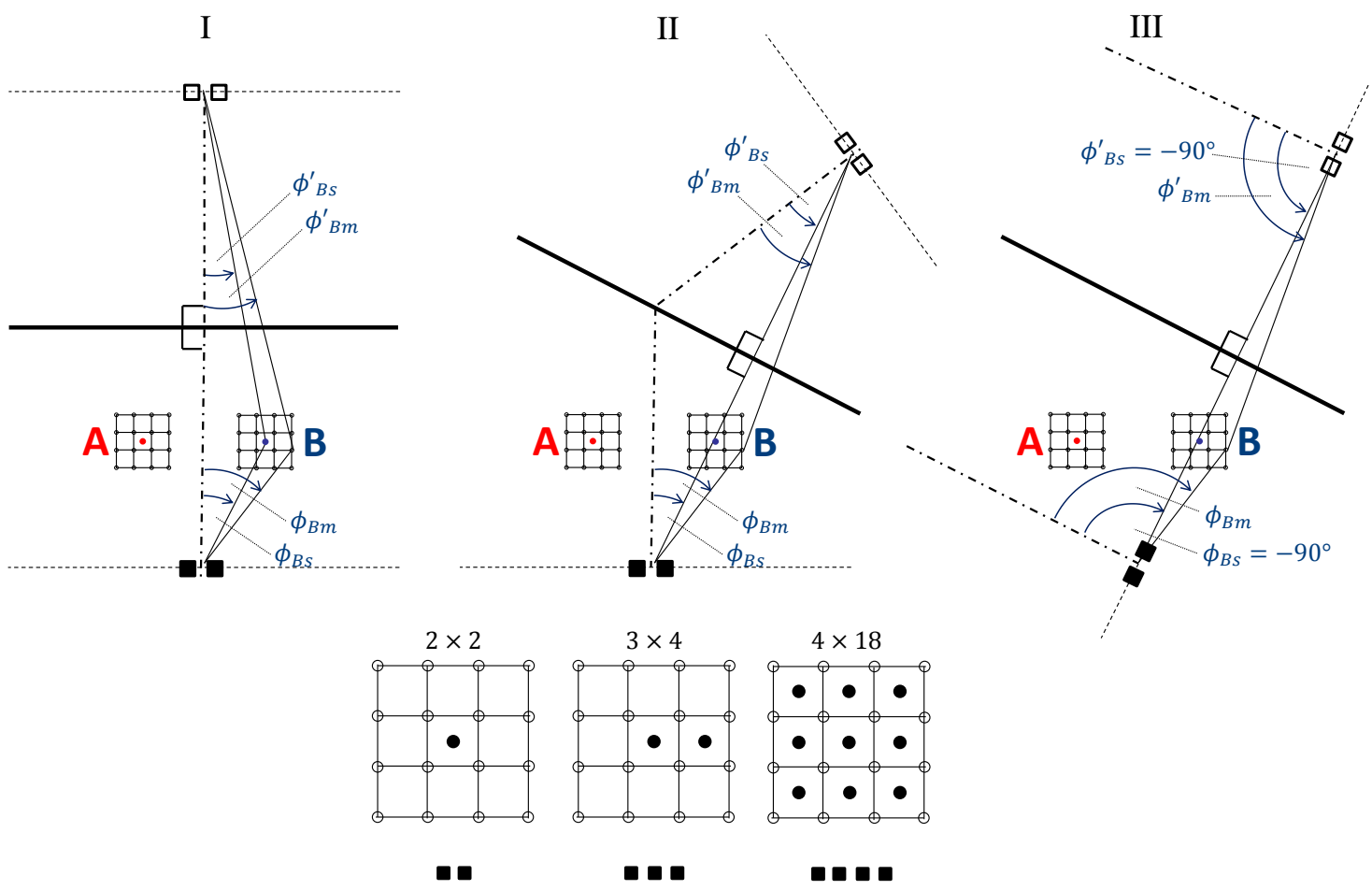

FIGURE 2: Top: Geometrical adjustments for a $2 \times 2$ system (not to scale): I: initial configuration, II: reorientation w.r.t. surface and III: reorientation w.r.t. surface and source rotation. Bottom: system expansion diagram for simulations. Symbol assignment: $\square$ source, $\square$ image source, $\bullet$ setup receiver, o monitor receiver, - surface.

\section{Simulation of Contrast and Effort Performance}

The geometries discussed above were tested in simulations for $2 \times 2,3 \times 4$ and $4 \times 18$ systems, with the source weights and receiver sound pressures calculated numerically. The expansion from $2 \times 2$ to larger systems is shown at the bottom of Fig. 2 . Each zone is controlled by $1(2 \times 2), 2$ $(3 \times 4)$ or $9(4 \times 18)$ setup receivers $(5 \mathrm{~cm}$ spacing). The performance in each zone is monitored with additional 16 receivers arranged on a square grid $(4 \times 4,5 \mathrm{~cm}$ spacing). Consistent monitoring across systems allows for meaningful comparisons of the results. Spacing between the sources is $d=5 \mathrm{~cm}$, which sets the aliasing frequency of the arrays at $3.4 \mathrm{kHz}$. The absorption coefficient of the surface is $\alpha=0.2$ across the whole frequency range, $250-3500 \mathrm{~Hz}$. The solution is regularized with a value of $\beta=10^{-5}$.

Contrast results for the $2 \times 2$ system are shown in Fig. 3 (a). The top and bottom figures show the contrast achieved under playback/setup conditions 1 and 2, respectively. As predicted, configurations II and III achieve contrast improvement w.r.t. configuration I (e.g., for configuration III the contrast is higher by $19 \mathrm{~dB}$ at $1.5 \mathrm{kHz}$ under condition 2). Compared to condition 1 , the depth of rapid contrast level fluctuations increases for configurations I and II under condition 2, which is attributed to the cosine function of the additional component $P_{B m C 2}$ in Eq. 7 (cf. Eq. 6). Physically, the fluctuations arise from the fact that under condition 2 the direct and reflected energies become more uniform in level, since the system is now trying to compensate for both sound field components (the cancellation of the direct sound deteriorates, whereas the cancellation of the reflection improves). The reflection remains low with respect to the direct sound for configuration II, giving lesser fluctuations in this case. An improvement of contrast in the low frequency range can be observed for configurations I and III under condition 2 . This is attributed to the influence of component $P_{B m C 3}$, which is not present in Eq. 6 for condition 1. As opposed to $P_{B m C 1}$ and $P_{B m C 2}, P_{B m C 3}$ is not related to the rapid contrast fluctuations (due to a much smaller argument of the cosine) and when it achieves negative values it counters the reflective components $P_{B m R 1}^{2}$ and $P_{B m R 2}^{2}$ (which are always positive). Component $P_{B m C 3}$ equalizes the room and 
its effectiveness depends on the geometrical relationship between the sources, zones and the surface. For configurations I and III, the component achieves negative values in the low frequency region, lowering Eq. 7's denominator and raising contrast.

The effort results for the $2 \times 2$ system under condition 2 are shown in Fig. 4 (a). As predicted, configuration III exhibits increased effort and the effort of configurations I and II is relatively low. The fluctuations in the effort curves are smallest for configuration III. This can be attributed to low $\Delta_{A s R 1}=\sin \phi_{A s}^{\prime}-\sin \phi_{B s}$, which reduces the significance of the combination term $P_{A s C 1}$ in the second term of Eq. 8. Physically, this means that there is little contribution of the reflection to the bright zone energy, in this case.

(a)
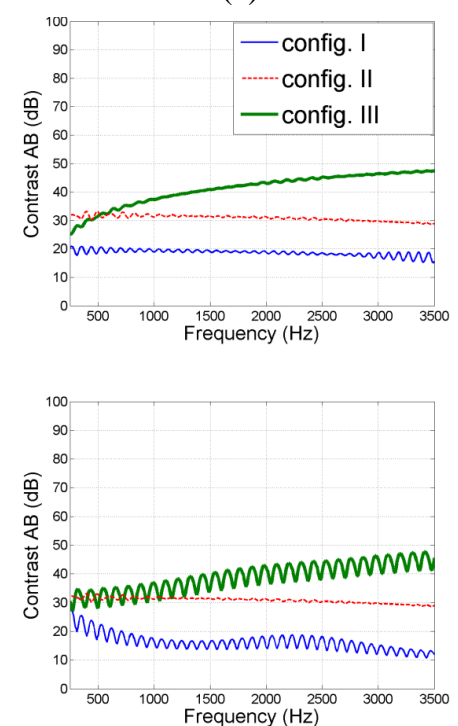

(b)
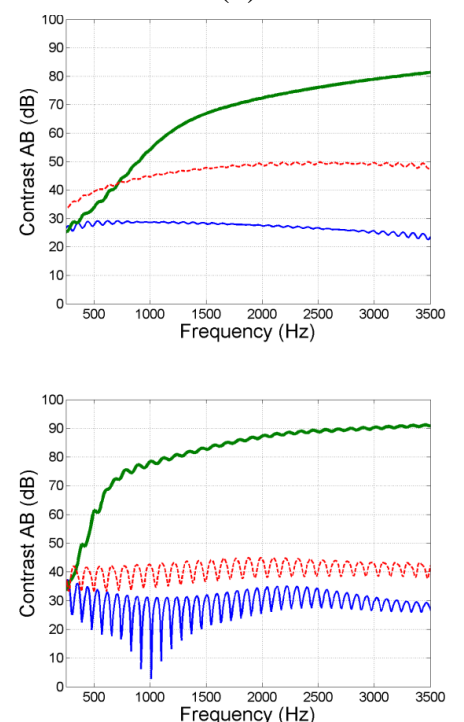

(c)
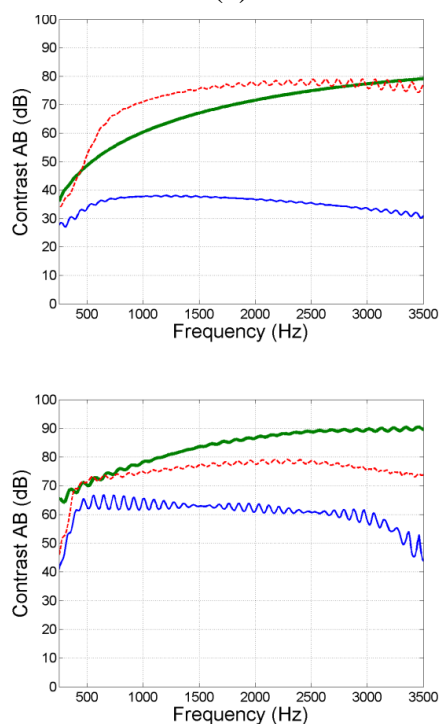

FIGURE 3: (Color online) Contrast results for (a) $2 \times 2$, (b) $3 \times 4$ and (c) $4 \times 18$ system. Top row - condition 1 , bottom row - condition 2. Solid line - configuration I, dashed line - configuration II, and thick solid line - configuration III.

(a)

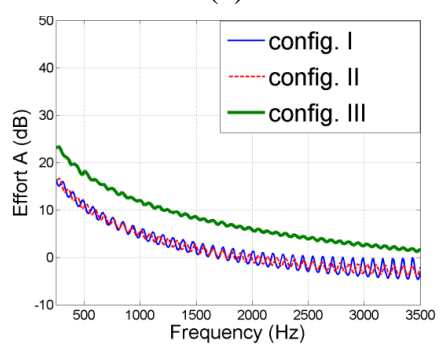

(b)

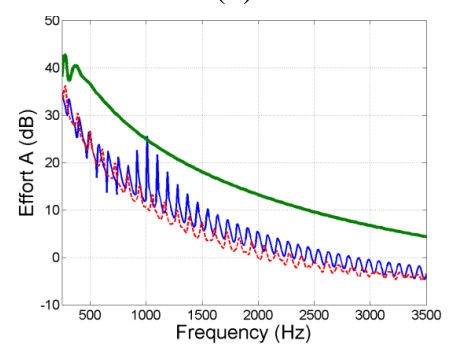

(c)

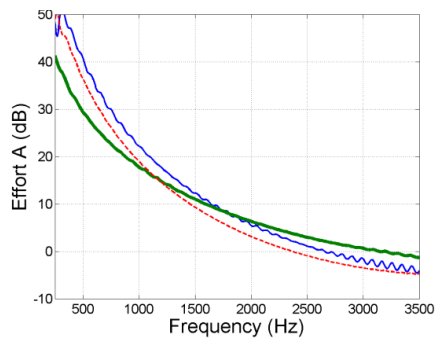

FigURE 4: (Color online) Effort results for (a) $2 \times 2$, (b) $3 \times 4$ and (c) $4 \times 18$ system, condition 2 . Solid line - configuration I, dashed line - configuration II, and thick solid line - configuration III.

The above results demonstrate that a substantial improvement in the contrast performance of a $2 \times 2$ system can be achieved through appropriate geometrical adjustment. The largest performance gains come at the cost of increased control effort. The setup conditions have a large influence on the system's contrast performance. Although applying condition 2 may result in improvement in the overall contrast level at low frequencies, the cancellation of the reflected sound is improved at the cost of the direct sound cancellation due to an insufficient number of degrees of freedom. This makes the fluctuations in the contrast level more profound as a result of the increased interaction between these two sound field components. The fluctuations are rooted in the comb filtering effect occurring for sound energies in both the bright and the dark zones, which may affect the perceived quality of the sound program reproduced in the bright zone, as well as 
the perceived suppression of sound in the dark zone [14]. Such considerations may have implications on the filter design procedure, in which the setup conditions may be chosen depending on the desired performance characteristics of the system: e.g. better contrast at low frequencies or reduced comb filtering.

The contrast performance of the $3 \times 4$ and $4 \times 18$ systems is shown in Fig. 3 (b) and (c), respectively. Similar trends in performance as in the $2 \times 2$ case are observed: optimized configurations achieve contrast gains w.r.t. the initial configuration (e.g. at $1.5 \mathrm{kHz}$ configuration III under condition 2 gains $51 \mathrm{~dB}$ and $21 \mathrm{~dB}$ with $3 \times 4$ and $4 \times 18$, respectively). In general, more fluctuations are observed under condition 2 , but better contrast is achieved at low frequencies. This shows that the design rules formulated using the analysis of the $2 \times 2$ performance are applicable to larger systems. The performance gain w.r.t. configuration I is particularly large for the $3 \times 4$ system, where configuration III reaches the level of performance similar to the $4 \times 18$ system, however with increased effort as shown in Fig. 4 (b) and (c). Moreover, for both $3 \times 4$ and $4 \times 18$ systems, configuration III achieves similar contrast under conditions 1 and 2 . This emphasizes the practical importance of geometrical optimization, which achieves the desired performance levels with systems of reduced size and when the playback room response is not available at the system setup stage.

\section{Conclusions}

The analysis presented above showed that geometrical parameters of a sound zone system, such as positioning of the sources with respect to zones and a reflecting surface, can have a large influence on the contrast performance of the ACC method. Based on the analysis of the contrast expressions for a $2 \times 2$ system, a way of mitigating the effect of reflection on contrast performance was suggested: the geometry of the system must be such that the array center, a setup receiver in the dark zone and the surface can be connected with a straight line perpendicular to the surface. Further improvement of performance was possible through the rotation of sources to endfire orientation w.r.t. the surface. The control effort cost of such adjustments was analyzed using expressions for $2 \times 2$ effort. Numerical simulations showed a large contrast performance gain for the optimized configurations and confirmed increased effort for the rotated array. The differences between the two setup conditions where also discussed, with possible implications on the filter design procedure. The scalability of the suggested optimization was demonstrated by the higher contrast of the simulated $3 \times 4$ and $4 \times 18$ systems. Similar levels of performance of the rotated array for both systems, and between two different setup conditions, indicated that the complexity of the optimized system can be reduced without significant loss of performance, even in situations where the playback room response is not available at the system setup stage. Further investigation of the relationship amongst source order, system conditioning, regularization and performance under reflective conditions is needed, especially for larger systems and higher-order reflections.

\section{REFERENCES}

[1] J.-W. Choi and Y.-H. Kim, "Generation of an acoustically bright zone with an illuminated region using multiple sources", J. Acoust. Soc. Am 111, 1695-1700 (2002).

[2] M. Shin, S. Lee, F. Fazi, P. Nelson, D. Kim., S. Wang, K.-H. Park, and J. Seo, "Maximization of acoustic energy difference between two spaces”, J. Acoust. Soc. Am. 128, 121-131 (2010).

[3] M. Jones and S. Elliott, "Personal audio with multiple dark zones", J. Acoust. Soc. Am. 124, 3497-3506 (2008). 
[4] Y. Wu and T. Abhayapala, "Simultaneous soundfield reproduction at multiple spatial regions", in Audio Engineering Society 128th Convention (2010).

[5] M. Poletti, "An investigation of 2-d multizone surround sound systems", in Audio Engineering Society 125th Convention (2008).

[6] J.-H. Chang and F. Jacobsen, "Sound field control with a circular double-layer array of loudspeakers”, J. Acoust. Soc. Am. 131, 4518-4525 (2012).

[7] M. Møller, M. Olsen, and F. Jacobsen, "A hybrid method combining synthesis of a sound field and control of acoustic contrast", in Audio Engineering Society 132nd Convention (2012).

[8] S. Elliott and M. Jones, "An active headrest for personal audio", J. Acoust. Soc. Am. 124, 2702-2709 (2006).

[9] F. Jacobsen, M. Olsen, M. Møller, and F. Agerkvist, "A comparison of two strategies for generating sound zones in a room", in 18th International Congress on Sound and Vibration (International Institute of Acoustics and Vibration) (2011).

[10] J.-H. Chang, C.-H. Lee, J.-Y. Park, and Y.-H. Kim, "A realization of sound focused personal audio system using acoustic contrast control”, J. Acoust. Soc. Am. 125, 2091-2097 (2009).

[11] S. Elliott, J. Cheer, H. Murfet, and K. Holland, "Minimally radiating sources for personal audio", J. Acoust. Soc. Am. 128, 1721-1728 (2010).

[12] M. Akeroyd, J. Chambers, D. Bullock, A. Palmer, A. Summerfield, P. Nelson, and S. Gatehouse, "The binaural performance of a cross-talk cancellation system with matched or mismatched setup and playback", J. Acoust. Soc. Am. 121, 1056-1069 (2007).

[13] J.-Y. Park, J.-H. Chang, and Y.-H. Kim, "Generation of independent bright zones for twochannel private audio system”, J. Audio Eng. Soc. 58, 382-393 (2010).

[14] H. Wittek, F. Rumsey, and G. Theile, "Perceptual enhancement of wavefield synthesis by stereophonic means”, J. Audio Eng. Soc. 55, 723-751 (2007). 